\title{
Preparation of Polypyrrole by Emulsion Polymerization Using Hydroxypropyl Cellulose
}

\author{
Masato AmAIKE ${ }^{1, \dagger}$ and Hiroyuki YAMAмото ${ }^{2}$ \\ ${ }^{1}$ R. \& D. Laboratory for High-performance Materials, Nippon Soda Co., Ltd., \\ 12-54, Goi-minamikaigan, Ichihara 290-0045, Japan \\ ${ }^{2}$ Institute of High Polymer Research, Faculty of Textile Science and Technology, \\ Shinshu University, Tokida 3-15-1, Ueda 386-8567, Japan
}

(Received December 27, 2005; Accepted March 14, 2006; Published June 9, 2006)

\begin{abstract}
Polypyrrole with particles of uniform diameter was easily obtained from an emulsion of hydroxypropyl cellulose (HPC) and pyrrole by chemical oxidative polymerization, using iron chloride as an oxidizing agent. The particle diameter of the polypyrrole could be controlled by changing the HPC concentration, and it was found that the preparation of water-dispersible polypyrrole was possible. The conductivity of the polypyrrole particles was of the order of $10^{-1} \mathrm{~S} / \mathrm{cm}$. [doi:10.1295/polymj.PJ2005198]

KEY WORDS Conducting Polymer / Polypyrrole / Hydroxypropyl Cellulose / Emulsion Polymerization / Conductivity / Control of Morphology /
\end{abstract}

Electrically conductive polymers such as polypyrrole may be prepared by electrolytic polymerization or chemical oxidative polymerization of a monomer. Polypyrrole can be obtained as a polymer with high electrical conductivity by polymerization under relatively mild conditions, and its electrical conductivity and structural stability are known to be excellent compared with other electrically conductive polymers. For these reasons, polypyrrole is the most extensively studied of these materials, and its development has been promoted in applications such as secondary batteries, ${ }^{1-7}$ capacitors, ${ }^{8,9}$ sensors, ${ }^{10-12}$ conductive fibers, ${ }^{13,14}$ and mechanical actuators. ${ }^{15,16}$

However, due to its strong intermolecular interactions, polypyrrole is generally insoluble and infusible, which has meant that its processability is poor and its field of use has been limited. ${ }^{17,18}$ Various experiments have been attempted in order to improve the processability and solubility of this material.

By synthesizing chemically modified monomers containing a linear alkyl group or carboxyl group at the 1-position or 3-position of the pyrrole ring, polypyrrole derivatives which are soluble in organic solvents have been obtained. ${ }^{19,20}$ However, there are still some problems associated with these materials. The synthesis of derivative monomers involves many steps. In addition, the conductivity of the resulting polypyrrole derivative is lower than that of the unsubstituted polypyrrole. With the use of sodium alkylbenzene sulfonate ${ }^{21}$ and sodium 2-ethylhexylsulfosuccinate $^{22}$ as dopants, solubilization into organic solvents was made possible because the bulkiness of the doped anion prevented aggregation of the polypyrrole chains. However, polypyrroles obtained by these methods were found to be soluble only in organic solvents, with low solubility, and were insoluble in aqueous systems.

Water-dispersible colloidal polypyrroles have been obtained by hybridization of a water-soluble polymer and an electrically conductive polymer. Numerous research groups have prepared sterically stabilized polypyrrole colloids using polymers such as poly(vinyl acetate),${ }^{23}$ chitosan, ${ }^{24}$ methyl cellulose, ${ }^{25}$ poly(ethylene oxide), ${ }^{26}$ and poly(vinyl pyrrolidone). ${ }^{27}$ The preparation of colloidal polypyrrole was also achieved by depositing polypyrrole on polystyrene latex ${ }^{28,29}$ or polybutylmethacrylate latex. ${ }^{30}$

In the application of electrically conductive polymers in devices, electrochemical properties such as electrical conductivity and redox properties must be taken into consideration, but morphology is also important. For example, when an electrically conductive polymer is used as the active material for a battery, it is necessary to increase the contact area between the electrode and electrolyte; in order to decrease the ion diffusion distance, it is also required that the active material be of small diameter and uniform size. ${ }^{26}$ Although polypyrrole containing particles of uniform diameter can be obtained from the colloidal polypyrroles described above, most of the materials used in this area are composites. Thus, the density of the active material in the prepared electrode is low.

In this paper, we report the preparation of a water-

${ }^{\dagger}$ To whom correspondence should be addressed (Fax: +81-436-21-9706, E-mail: m.amaike@nippon-soda.co.jp). 
(a)

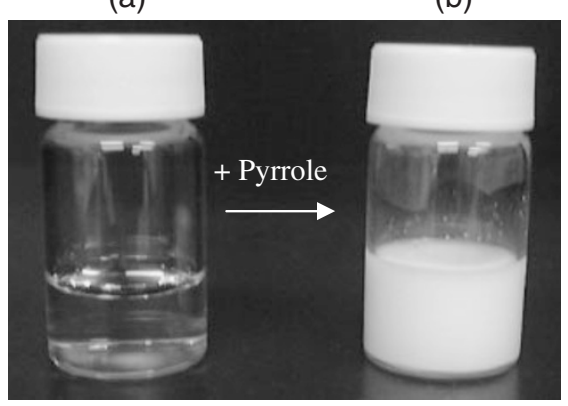

(c)

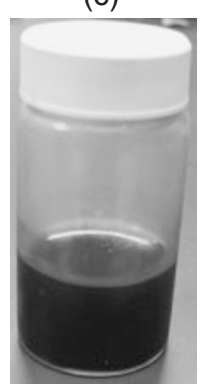

Figure 1. Photographs of (a) $5 \mathrm{wt} \%$ HPC aqueous solution, (b) pyrrole $+5 \mathrm{wt} \%$ HPC emulsion, and (c) polypyrrole dispersion. $[$ Pyrrole $]=0.6 \mathrm{M} .\left[\mathrm{FeCl}_{3}\right]=1.9 \mathrm{M}$.

dispersible polypyrrole by polymerization of an emulsion of hydroxypropyl cellulose (HPC) and pyrrole monomer. Polypyrrole containing particles of uniform diameter may be easily obtained using this method. In addition, HPC, which was used as a steric stabilizer in the emulsion polymerization process, was found only in insignificant amounts in the resulting polypyrrole particles.

\section{EXPERIMENTAL}

\section{Materials}

Pyrrole was purchased from Tokyo Kasei Kogyo Co., Ltd. Before use, vacuum distillation was conducted using calcium hydride $\left(\mathrm{CaH}_{2}\right)$. HPC (HPC-SL, MW 77122), was obtained from Nippon Soda Co., Ltd. Calcium hydride and iron chloride hexahydrate, which is an oxidizing agent for pyrrole, were purchased from Wako Pure Chemical Industries, Ltd. The molecular weight cut-off of the cellulose dialysis tube was 12000-14000.

\section{Preparation of Polypyrrole Colloids}

Various amounts of HPC were dissolved in $10 \mathrm{~mL}$ of water to obtain solutions of predetermined concentrations. To each of these HPC aqueous solutions was added, with stirring, $0.4 \mathrm{~g}(6.0 \mathrm{mmol})$ of pyrrole, and the mixture was stirred well and cooled to $0{ }^{\circ} \mathrm{C}$. $\mathrm{FeCl}_{3} \cdot 6 \mathrm{H}_{2} \mathrm{O}(5.03 \mathrm{~g}, 18.6 \mathrm{mmol})$ was dissolved in $3 \mathrm{~mL}$ of water, and, using an ice bath, this solution was slowly added, with stirring, to the HPC-pyrrole mixed solution. Stirring was continued for $6 \mathrm{~h}$, with the temperature maintained in the range $0-5^{\circ} \mathrm{C}$. After completion of the reaction, the solution was dialyzed with a cellulose dialysis tube, followed by centrifuging (10,000 rps) for $20 \mathrm{~min}$ and water dispersion, each of which were conducted three times. Thus, $\mathrm{FeCl}_{2}$, unreacted $\mathrm{FeCl}_{3}$ and $\mathrm{HPC}$ were removed, and a black solution was obtained.

To measure the viscosity of the solutions, a Sinewave Vibro Viscometer SV-10 (A\&D Company,
Ltd.) was used. A particle size detector (Malvern Instruments, model HPPS-ET) was used for particle size measurements. The structure of the polypyrrole product was confirmed using FT-IR. Conductivity was determined, by a four-point probe method, for a pellet obtained by pressing dried polypyrrole powder. FT-IR spectra were obtained with a Nicolet MEGA IR 760 (Nicolet) (KBr pellet, $0.1 \mathrm{wt} \%$ polypyrrole). Elemental analysis was conducted using an Elementar Model Vario EL III CHNSO elemental analyzer and a Dia Instruments Model TOX-100 Sulfur/Chlorine analyzer. For morphological observations of polypyrrole, SEM (JEOL, model JXA-840) and TEM (Hitachi, model H-7100FA) were used.

\section{RESULTS AND DISCUSSION}

\section{Preparation of Pyrrole-HPC Emulsion}

Transparent homogeneous solutions of HPC (1.0, 5.0, 10, and $20 \mathrm{wt} \%$ ) were prepared by dissolving HPC in distilled water that had been bubbled with nitrogen for $15 \mathrm{~min}$ [Figure 1(a)]. Dropwise addition of pyrrole to these aqueous solutions resulted in emulsification [Figure 1(b)]; as a result, a large change in viscosity was observed for mixed solutions with an HPC concentration of more than $10 \mathrm{wt} \%$ (Table I). The HPC aqueous solution was highly viscous, but its viscosity decreased to less than $1 / 10$ of its original value after the addition of pyrrole due to emulsification.

In solutions with low HPC concentrations, changes in viscosity were not observed even when emulsions were formed. However, changes in the particle diameter of HPC were observed in particle-size distribution measurements, for which a dynamic light-scattering method was used. When the HPC concentration was $1 \%$, the addition of pyrrole resulted in the formation of an emulsion, and the particle diameter was observed to decrease from $2500 \mathrm{~nm}$ to $539 \mathrm{~nm}$. This phenomenon may be explained as follows: in the absence of pyrrole, the molecular chain of HPC is stretched, 
Table I. Viscosity and average particle diameter of HPC-pyrrole monomer emultion, and the effect of varying HPC concentration on conductivity and particle diameter of the resulting polypyrrole

\begin{tabular}{|c|c|c|c|c|}
\hline & \multicolumn{4}{|c|}{ HPC concentration/wt $\%$} \\
\hline & 1.0 & 5.0 & 10 & 20 \\
\hline \multicolumn{5}{|l|}{ I) HPC aqueous solution } \\
\hline Viscosity $/ \mathrm{mPa} \cdot \mathrm{s}$ & 2.32 & 16.4 & 131 & 860 \\
\hline Diameter $^{\mathrm{b}} / \mathrm{nm}$ & 2542 & 4363 & $>6000$ & $>6000$ \\
\hline \multicolumn{5}{|l|}{ II) $\mathrm{HPC}+$ Pyrrole $^{\mathrm{c}}$ emulsion } \\
\hline Viscosity $/ \mathrm{mPa} \cdot \mathrm{s}$ & 2.26 & 15.2 & 76.2 & 76.0 \\
\hline Diameter ${ }^{\mathrm{b}} / \mathrm{nm}$ & 539 & 4395 & $>6000$ & $>6000$ \\
\hline$(\text { Pyrrole })^{c} /(\text { HPC unit })^{d}$ mole ratio & 22.1 & 4.42 & 2.22 & 1.11 \\
\hline \multicolumn{5}{|c|}{ Polypyrrole prepared in HPC solutions } \\
\hline III) Formation & precipitate & $\begin{array}{l}\text { colloidal } \\
\text { dispersion }\end{array}$ & gel & gel \\
\hline III) Pellet conductivity $/ \mathrm{S} \cdot \mathrm{cm}^{-1}$ & $2.0 \times 10^{-1}$ & $1.2 \times 10^{-1}$ & $4.4 \times 10^{-3}$ & $1.3 \times 10^{-4}$ \\
\hline VI) Particle size range ${ }^{\mathrm{f}} / \mathrm{nm}$ & $500 \pm 40$ & $130 \pm 25$ & - & - \\
\hline
\end{tabular}

${ }^{\text {a }}$ Measured at $20^{\circ} \mathrm{C}$. ${ }^{b}$ Determined by dynamic light scattering at $20^{\circ} \mathrm{C}$. ${ }^{c}$ Pyrrole monomer concentration was $0.6 \mathrm{~mol} \mathrm{dm}^{-3}$. ${ }^{\mathrm{d}}$ Mole ratios of pyrrole monomer to HPC unit (MW 368). ${ }^{\mathrm{e}}$ Determined by the four-point probe method on compressed pellets at room temperature. ${ }^{\mathrm{f}}$ Determined by SEM and TEM.

but when an emulsion is formed due to the addition of pyrrole, the molecular chain becomes spherical. In the absence of pyrrole, an HPC solution with a concentration of 5\% exhibited a unimodal particle distribution with a peak at $4363 \mathrm{~nm}$. The solution was observed to become cloudy when an emulsion was formed on addition of pyrrole; however, the particle distribution diagram, with a peak at $4395 \mathrm{~nm}$, changed very little. This indicates the presence of unreacted HPC, which was not involved in emulsification. With an HPC concentration of $10 \%$, the particle diameter measurements for HPC before addition of pyrrole gave a result of more than $6 \mu \mathrm{m}$, which was beyond the measurement limit.

\section{Preparation of Water-Dispersible Polypyrrole}

An oxidizing agent, iron chloride, was added to the emulsified pyrrole-HPC solution to initiate pyrrole polymerization. The entire solution swiftly turned black.

When the HPC concentration was $5 \mathrm{wt} \%$, the black reaction solution contained no precipitate. In order to remove excess iron chloride, inorganic by-products $\left(\mathrm{FeCl}_{2}, \mathrm{HCl}\right.$, etc.), and $\mathrm{HPC}$, the black solution was dialyzed and centrifuged, and the resulting sediment was redispersed in water. These centrifugation and redispersion steps were carried out several times until a neutral $\mathrm{pH}$ was obtained, resulting in a black solution of polypyrrole (polypyrrole colloid solution) [Figure 1(c)].

For an HPC concentration of $1 \mathrm{wt} \%$, the reaction solution contained a black precipitate. The mixture was filtered, and the precipitate was washed several times with water and methanol and then dried under vacuum to obtain a black powder. For HPC concentra- tions of 10 and $20 \mathrm{wt} \%$, the reaction solution formed a gel $1 \mathrm{~h}$ after the addition of iron chloride. This may have been due to entangling of the polypyrrole chain with the HPC molecular chain as polypyrrole was formed.

\section{IR Spectra of Polypyrrole}

IR absorption spectra for HPC, pure polypyrrole, and four HPC-polypyrrole composites are shown in Figure 2. In all spectra, absorption bands due to the polypyrrole ring structure $\left[1529 \mathrm{~cm}^{-1}(v\right.$ ring: antisymmetric), $1443 \mathrm{~cm}^{-1}$ ( $v$ ring: symmetric), and 1289 $\mathrm{cm}^{-1}$ ( $v$ ring: $\mathrm{C}-\mathrm{C}, \mathrm{C}-\mathrm{N}, \mathrm{C}=\mathrm{C}$ )] were identified. ${ }^{31,32}$ The IR spectra for polypyrrole prepared in $1 \mathrm{wt} \%$ and $5 \mathrm{wt} \%$ HPC solutions $(\mathrm{b}, \mathrm{c})$ generally matched the IR spectrum of polypyrrole without HPC (a). The fact that an absorption band due to HPC was not observed implies that the amounts of HPC present in the polypyrrole were relatively small. Because HPC does not act as a dopant, it is thought that HPC is adsorbed onto the polypyrrole surface rather than forming a composite with the polypyrrole molecule. In contrast, the IR spectra for the polypyrrole gel prepared using $10 \mathrm{wt} \%$ and $20 \mathrm{wt} \%$ HPC solutions (d, e) show absorption bands $\left[3451 \mathrm{~cm}^{-1}(v \mathrm{OH}), 2972 \mathrm{~cm}^{-1}(v \mathrm{C}-\right.$ $\mathrm{H})$ ] due to HPC. The strength of the absorption bands became greater as the HPC concentration of the original solution increased. Thus, it was confirmed that the hydrogel consisted of polypyrrole and HPC.

\section{Elemental Analysis of Polypyrrole}

The results of elemental analysis of the polypyrrole samples prepared with HPC solutions of various concentrations are shown in Table II.

A control sample containing no HPC yielded a C:N 


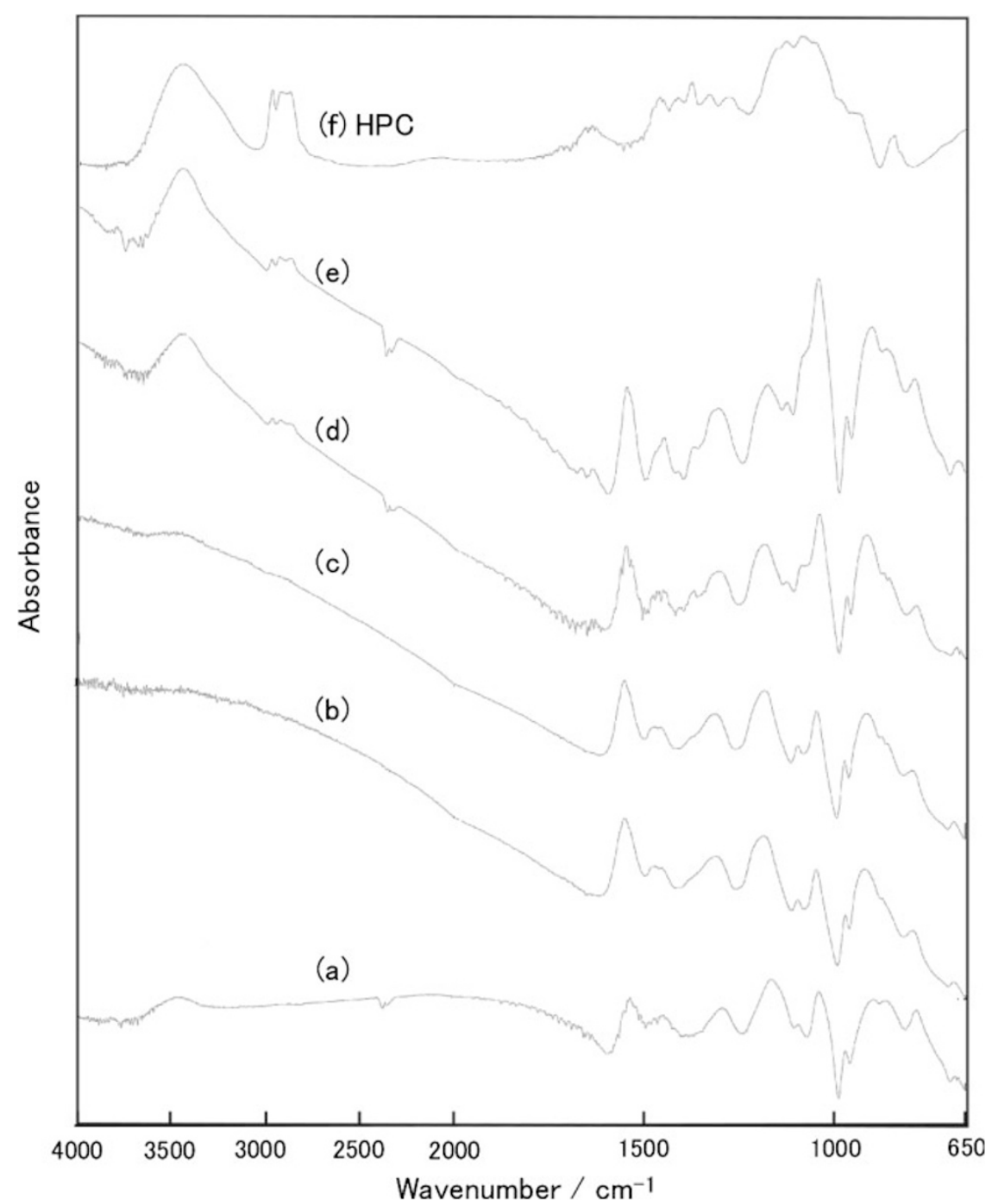

Figure 2. FT-IR spectra of (a-e) polypyrrole prepared using HPC solutions of different concentrations and (f) pure HPC. The concentrations of HPC in the aqueous polymerization solutions were (a) none, (b) 1.0, (c) 5.0, (d) 10, and (e) $20 \mathrm{wt} \%$.

Table II. Elemental analysis of polypyrrole samples prepared at different initial HPC concentrations

\begin{tabular}{lrrrccc}
\hline & \multicolumn{1}{c}{$\mathrm{C}$} & \multicolumn{1}{c}{$\mathrm{H}$} & $\mathrm{N}$ & $\mathrm{O}$ & $\mathrm{Cl}$ & $\mathrm{Fe}$ \\
\hline PPy (without HPC) & 4.03 & 3.19 & 1.00 & 0.44 & 0.34 & 0.001 \\
PPy (1 wt \% HPC) & 4.81 & 4.28 & 1.00 & 0.83 & 0.25 & 0.005 \\
PPy (5 wt \% HPC) & 5.12 & 5.29 & 1.00 & 0.97 & 0.23 & 0.006 \\
PPy (10 wt \% HPC) & 7.38 & 9.44 & 1.00 & 2.57 & 0.28 & 0.009 \\
PPy (20 wt \% HPC) & 12.83 & 19.22 & 1.00 & 5.44 & 0.28 & 0.005 \\
\hline
\end{tabular}

ratio of 4.0:1, as expected for polypyrrole. The results demonstrate the difference between the theoretical polypyrrole structure $\left(\mathrm{C}_{4} \mathrm{H}_{3} \mathrm{~N}\right)_{\mathrm{n}}$ and synthesized polypyrrole. Oxygen is present in polypyrrole in the form of $\mathrm{C}-\mathrm{O}, \mathrm{C}-\mathrm{OH}$ and $\mathrm{C}=\mathrm{O}$ bonds. ${ }^{33,34}$ These bonds may be formed during the polymerization process as a consequence both of the presence of water in the solution and of the reaction of atmospheric oxygen with polypyrrole. The $\mathrm{Cl}$ anions act as a doping agent. ${ }^{35}$ The presence of a small amount of $\mathrm{Fe}$ is due to the use of $\mathrm{FeCl}_{3}$ as an oxidation agent.
The results of elemental analysis of the polypyrrole powder and colloid samples prepared in $1 \mathrm{wt} \%$ and $5 \mathrm{wt} \%$ HPC solutions were different from those obtained for the bulk polypyrrole powder, indicating the presence of HPC. The reason this was not observed in the IR spectra may be that the intense bands of the polypyrrole, which is a strong IR absorber, ${ }^{36}$ submerged the HPC bands. The polypyrrole gels prepared in $10 \mathrm{wt} \%$ and $20 \mathrm{wt} \%$ HPC solutions show higher $\mathrm{C}, \mathrm{H}, \mathrm{O}$ ratios relative to those of powder and colloid polypyrroles, which supports the FT-IR results.

\section{Conductivity of Polypyrrole}

The obtained polypyrroles were in a doped state, and exhibited electrical conductivity. To measure the conductivity of the polypyrrole obtained, powder samples were made into pellets, and the four-point probe method was applied. Polypyrrole colloid and gel were cast on a glass board, dried, and made into powders, 
(a)

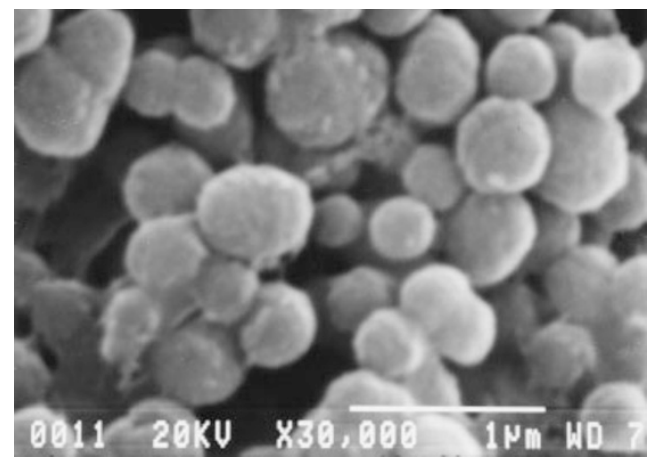

(b)

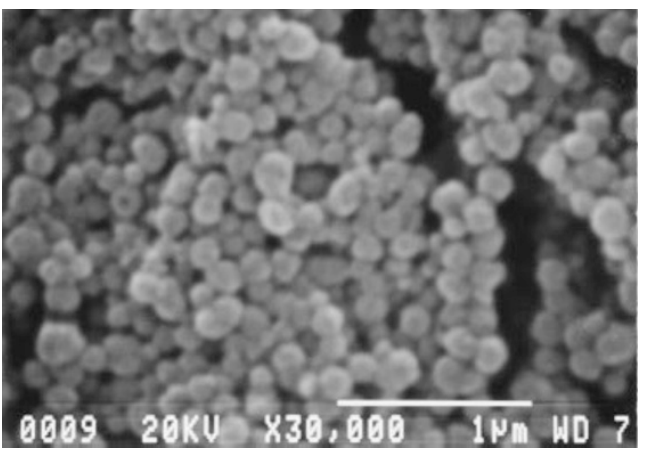

(c)

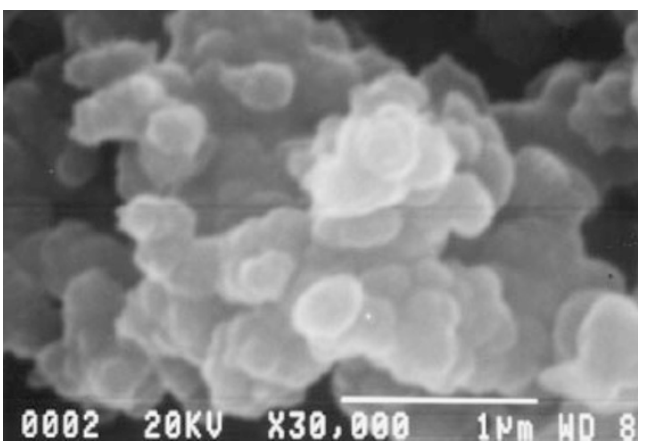

Figure 3. SEM micrographs of polypyrrole prepared in HPC solutions of different concentrations. The concentrations of HPC in the aqueous polymerization solutions were (a) 1.0, (b) $5.0 \mathrm{wt} \%$, and (c) none.

and their conductivities were measured in the same way as for the first powder sample.

The pellet conductivity of the polypyrrole colloid was found to be $1.2 \times 10^{-1} \mathrm{~S} / \mathrm{cm}$, which was comparable to that of the powder (Table I). The pellet conductivity of the gel was lower than those of the powder and colloid $\left(10^{-4} \sim 10^{-3} \mathrm{~S} / \mathrm{cm}\right)$. This may be due to the presence of large amounts of HPC in the gel, so that the HPC chain disturbs the contact between polypyrrole chains, causing a decrease in conductivity.

\section{Morphology of Polypyrrole Colloid}

Figures 3 and 4 show SEM and TEM micrographs of the polypyrrole colloid and powder particles. These were used to determine particle diameters, which are shown in Table I. Both types of particle (colloid and powder) were spherical. However, the polypyrrole (a)

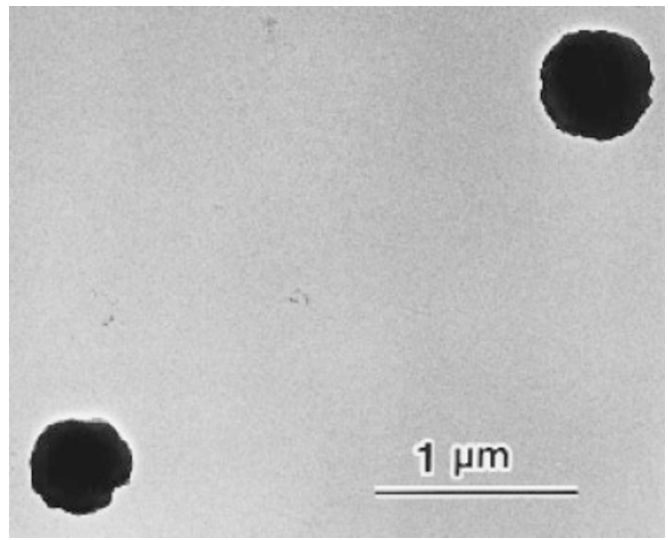

(b)

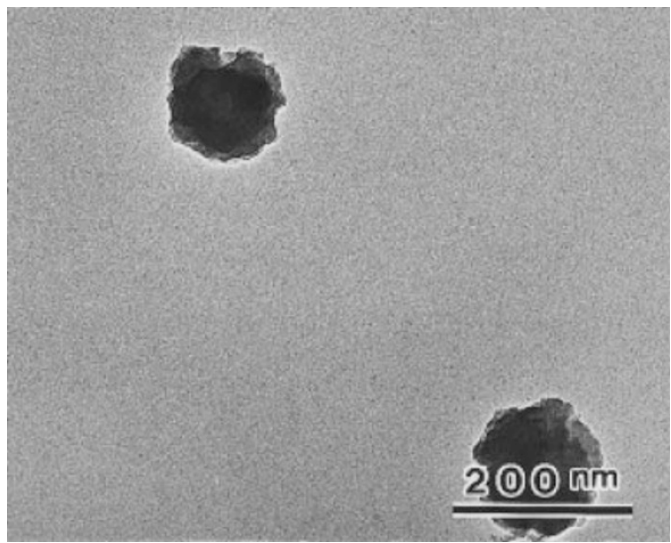

Figure 4. TEM micrographs of polypyrrole prepared in HPC solutions of different concentrations. The concentrations of HPC in the aqueous polymerization solutions were (a) 1.0 and (b) $5.0 \mathrm{wt} \%$.

particle sizes were found to vary depending upon HPC concentration. HPC was not observed in the TEM micrographs shown in Figure 4; only polypyrrole was found to be present.

The diameters of colloid particles obtained by polymerization in $5 \mathrm{wt} \%$ HPC solution were small (140 \pm $30 \mathrm{~nm}$ ) [Figure 4(b)]. Powder particles obtained from $1 \mathrm{wt} \%$ HPC solution were spherical, with diameters of $500 \pm 40 \mathrm{~nm}$ [Figure 4(a)]. In contrast, polypyrrole particles prepared in the same way but without HPC were found to be non-uniform, non-spherical, and chain-like [Figure 3(c)]. ${ }^{6,29}$

A TEM micrograph of the polymerization reaction mixture containing pyrrole and $1 \mathrm{wt} \% \mathrm{HPC}$ solution is shown in Figure 5. Here, we can see the process of formation of polypyrrole particles; polypyrrole and HPC are present in a cluster. The following polypyrrole formation process may be suggested: initially, an emulsion is formed by the pyrrole and HPC due to a hydrophilic interaction between the hydroxyl groups associated with the ether moieties of HPC and water, and a hydrophobic interaction between the pyrrole monomer and the HPC main chain; ${ }^{37}$ polymerization then occurs in this state. HPC prevents fur- 


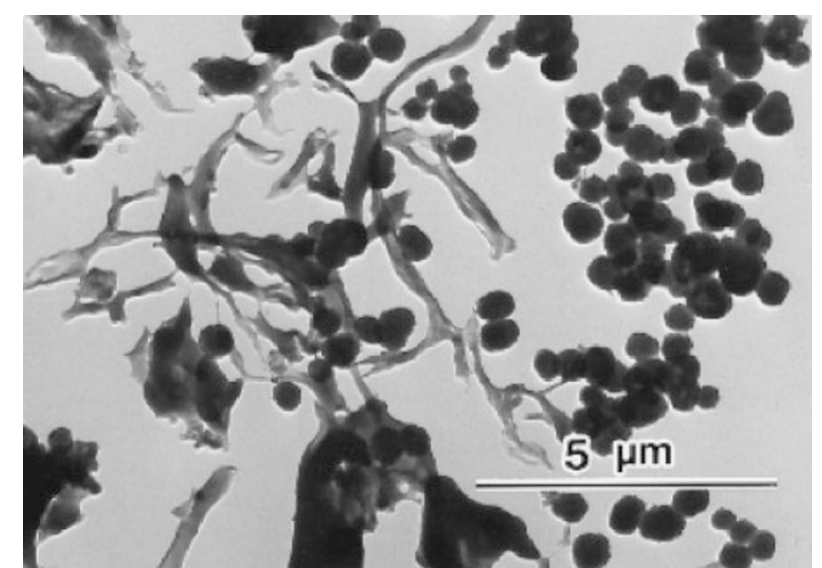

Figure 5. TEM micrograph of the reaction mixture containing polypyrrole and $1.0 \mathrm{wt} \% \mathrm{HPC}$.

ther aggregation by providing steric stabilization during the polymerization, so that spherical particles are obtained. The polypyrrole particles formed are in an oxidized state and have a cationic charge. For this reason, they do not form a connection with the non-ionic HPC, and gradually become separate. The resulting polypyrrole particles contain a small amount of HPC.

Thus, it was found that HPC concentration controls the shape and size of the polypyrrole particles, and that the amount of HPC present during the reaction has a significant influence on the shape of the resulting particles. It was also found that the formation of an HPC-pyrrole emulsion plays an important role in the formation of polypyrrole particles with uniform diameters.

\section{CONCLUSION}

Polypyrrole particles with uniform diameters were obtained by chemical oxidative polymerization of an HPC-pyrrole emulsion. By changing the HPC concentration, the particle diameter of the obtained polypyrrole could be controlled, and water-dispersible polypyrrole was obtained. The resulting polypyrrole particles show conductivity of the order of $10^{-1} \mathrm{~S} / \mathrm{cm}$. Because polymerization takes place in a water-based system without organic solvent, it is less expensive than other polymerization methods and ecologically ideal.

In the future, we plan to prepare electrodes from the resulting polypyrrole, study its electrical properties, and apply the material in lithium secondary batteries.

\section{REFERENCES}

1. S. Panero, P. Prosperi, and B. Scrosati, Electrochim. Acta., 32, 1465 (1987).

2. T. Osaka, K. Naoi, and S. Ogano, J. Electrochem. Soc., 135, 1071 (1988).
3. K. Nishio, M. Fujimoto, N. Yoshinaga, N. Furukawa, O. Ando, H. Ono, and T. Suzuki, J. Power Sources, 34, 153 (1991).

4. R. C. D. Peres and M.-A. De Paoli, J. Power Sources, 40, 299 (1992).

5. T. Osaka, T. Momma, K. Nishimura, S. Kakuda, and T. Ishii, J. Electrochem. Soc., 141, 1994 (1994).

6. K. Nishio, M. Fujimoto, O. Ando, H. Ono, and T. Murayama, J. Appl. Electrochem., 26, 425 (1996).

7. T. Osaka, T. Momma, H. Ito, and B. Scrosati, J. Power Sources, 68, 392 (1997).

8. Y. Kudoh, S. Tsuchiya, T. Kojima, M. Fukuyama, and S. Yoshida, Synth. Met., 41-43, 1133 (1991).

9. L. H. M. Krings, E. E. Havinga, and J. J. T. M. Donkers, Synth. Met., 54, 453 (1993).

10. C. G. J. Koopal, M. C. Feiters, and R. J. M. Nolte, Bioelectrochem. Bioenerg., 29, 159 (1992).

11. M. J. Swann, D. Bloor, T. Haruyama, and M. Aizawa, Biosens. Bioelectron., 12, 1169 (1997).

12. B. Deore, Z. Chen, and T. Nagaoka, Anal. Chem., 72, 3989 (2000).

13. R. V. Gregory, W. C. Kimbrell, and H. H. Kuhn, Synth. Met., 28, C823 (1989).

14. H. H. Kuhn, A. D. Child, and W. C. Kimbrell, Synth. Met., 71, 2139 (1995).

15. E. Smela, O. Inganäs, and I. Lundström, Science, 268, 1735 (1995).

16. R. H. Baughman, Synth. Met., 78, 339 (1996).

17. S. Rapi, V. Bocchi, and G. P. Gardini, Synth. Met., 24, 217 (1988).

18. "Conjugated Polymers and Related Materials," R. Qian and B. Rånby, Ed., Oxford Univ. Press, London, 1993.

19. J. Rühe, T. A. Ezquerra, and G. Wegner, Synth. Met., 28, C117 (1989).

20. I. T. Kim and R. L. Elsenbaumer, Synth. Met., 84, 157 (1997).

21. J. Y. Lee, D. Y. Kim, and C. Y. Kim, Synth. Met., 74, 103 (1995).

22. K. S. Jang, S. S. Han, J. S. Shu, and E. J. Oh, Synth. Met., 119, 107 (2001).

23. S. P. Armes, J. F. Miller, and B. Vincent, J. Colloid Interface Sci., 118, 410 (1987).

24. E. Khor and J. L. H. Whey, Carbohydr. Polym., 26, 183 (1995).

25. F. Epron, F. Henry, and O. Sagnes, Makromol. Chem., Macromol. Symp., 35/36, 527 (1990).

26. O. Inganäs, Br. Polym. J., 20, 233 (1988).

27. H. Shiigi, M. Kishimoto, H. Yakabe, B. Deore, and T. Nagaoka, Anal. Sci., 18, 41 (2002).

28. A. Yassar, J. Roncali, and F. Garnier, Polym. Commun., 28, 103 (1987).

29. S. F. Lascells, S. P. Armes, P. A. Zhdan, S. J. Greaves, A. M. Brown, J. F. Watts, S. R. Leadley, and S. Y. Luk, J. Mater. Chem., 7, 1349 (1997).

30. F. M. Hujis, J. Lang, D. Kalicharan, F. F. Vercauteren, J. J. L. van der Want, and G. Hadziioannou, J. Appl. Polym. Sci., 79, 900 (2001).

31. J. T. Lei, Z. Cai, and C. R. Martin, Synth. Met., 46, 53 (1992). 
32. J. Mikat, I. Orgzall, and H. D. Hochheimer, Synth. Met., 116, 167 (2001).

33. J. C. Thiéblemont, J. L. Gabelle, and M. F. Planche, Synth. Met., 66, 243 (1994).

34. N. Toshima and O. Ihata, Synth. Met., 79, 165 (1996).
35. S. Machida and S. Miyata, Synth. Met., 31, 311 (1989).

36. S. F. Lascelles and S. P. Armes, J. Mater. Chem., 7, 1339 (1997).

37. Y. K. Yarovoy and M. M. Labes, J. Am. Chem. Soc., 119, 12109 (1997). 